\title{
Noise Estimation using Kalman Filter in Image Restoration
}

\author{
B. Nagasirisha, V.V.K.D.V. Prasad
}

\begin{abstract}
Medical image processing plays a vital role in medical sciences from the past decades. Medical image processing becomes simple and useful with the advancement of image processing techniques. Medical images are used to observe the information related to inside the organs of human body. For better diagnoses and analysis of disease the image should be clear, noise free and more informative also. Usually medical images are corrupted by different noises in image acquisition and transmission process. The basic challenge in medical image processing is noise removal without losing diagnostic information. Image restoration is the one of the technique to recover the original image from the degraded image. In this paper, we are proposing a kalman filter to estimate the noise function from the degraded image and to reconstruct the original image. Here we are taking into account that the medical image was corrupted by the gaussian, speckle and salt \& pepper noise. The simulation result infers that the proposed blind deconvolution method can be able to suppress the noise well and also preserve edge information without losing diagnostic data.
\end{abstract}

Index Terms: speckle noise, salt and pepper noise, Kalman filter.

\section{INTRODUCTION}

From the decades onwards the continuous research and improvement observed in digital image processing techniques. This leads to image processing becomes simple, plays a key role in medical research, medical education and clinical routine. Modern image processing techniques are more useful to improve the clinical services like diagnosis of disease and in surgical issues. The diagnoses of disease become more accurate with the use of medical pictures such as Computer Topography (CT), Magnetic resonance imaging (MRI), X-ray images, Magnetic resonance spectroscopy image (MRSI), Optical surface scan (OSS) and fluorescence endoscopy. The most frequently used process in medical image processing is image restoration. Image restoration is used to enhance the image quality and also preserves the diagnostic data. Image restoration is similar to the image enhancement but restoration is objective in nature and enhancement is subjective in nature. The restored image quality is assessed quantitatively but the enhanced image quality is assessed by human perception.

Image restoration is the technique to recover the original image from the noise corrupted image. Image restoration

Revised Manuscript Received on August 20, 2019.

B. Nagasirisha*, ECE Department, Gudlavalleru Engineering College, Gudlavalleru, India.

V.V.K.D.V. Prasad, ECE Department, Gudlavalleru Engineering College, Gudlavalleru, India. popular and useful in many applications. Image processing

process [4][5] carried by two stages, one is image degradation function measurement stage and reconstruction of degraded image using filter is another stage..

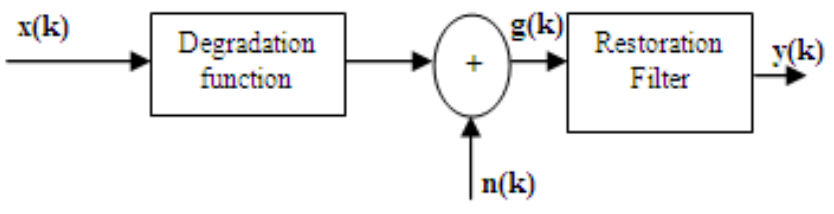

Fig 1.Image restoration model

In Fig 1. the test image is represented by the $\mathrm{x}(\mathrm{k}), \mathrm{n}(\mathrm{k})$ is the unknown noise adding to the image, resulting noisy image mentioned by $\mathrm{g}(\mathrm{k})$, and $\mathrm{y}(\mathrm{k})$ is the restored image. Image restoration method is the inverse in process because degradation function is measured or observed from the test image and applies it in reverse manner on degraded image to get the original image. Usually the degradation function either to be observed from the test image or estimated by the mathematical model. Normally image restoration process done in spatial domain and frequency domain. In spatial domain restoration filtering operation performed directly on pixels. So, spatial domain restoration method performance primarily depends on image intensity level. Image restoration methods are categorized as non blind deconvolution and blind deconvolution restoration techniques. These techniques are spatial domain techniques. The conventional non blind restoration methods like inverse filter, wiener filter, and Richardson-Lucy techniques using the priori known point spread function (PSF) or degradation function to recover the original image. In many real and practical cases it is not possible to get the degradation function easily. The method of reconstruction of the image with unknown degradation function is represented as blind deconvolution restoration. The blind deconvolution restoration is the process of estimation of the PSF in simultaneous with the reconstruction of original image. The non blind deconvolution restoration methods are suitable in real time medical image processing applications because these techniques has no need of prior information about image and noise.

In this paper we are proposing a kalman filter to remove the unknown channel noise and to avoid the use of known PSF in degradation model. The kalman filter [1] is a linear estimator used to minimize the MSE between the estimated and the desired steps. 


\section{NOISE IN MEDICAL IMAGES}

Usually medical images are affected by the different noises in acquisition and transmission stages. The medical images are generally corrupted by the distinct noises like periodic noise, Gaussian noise (additive noise), random noise, salt \&pepper noise (Impulse noise), and speckle noise (multiplicative noise).Gaussian noise [6][7] is generally considered as a common electronic noise. Mainly in medical image acquisition stage images are corrupted by the Gaussian noise. Gaussian noise is additive in nature. Additive noises are easily modeled and removed in medical images but sometimes its affect is more. Gaussian noise effect can be more observed in high quality medical images as blurring edges. Commonly wiener filter is used to obtain an optimal estimate but the drawback of the wiener filter is if initial estimation is not correct the process will never converges.

If the image which is contaminated by bright pixels at dark side of the image and dark pixels at bright side of the image, such type of noise in image referred as salt and pepper noise. The salt and pepper noise occurs in medical images because of analog to digital conversion of image and transmission process. The salt and pepper noise is also referred as impulsive noise and it is efficiently removed from the image by the median filter and morphological filters. Image restoration using median filter needs a known linear degradation function to know the degradation model. But in image restoration noise removal is not only the criteria that should also gives the desired restoration with mathematical goodness.

Commonly medical images are degraded by the speckle noise in medical image acquisition stage. Usually it is a Signal dependent noise or granular noise. Speckle noise in image can't be modeled easily because noise is convolved with image directly. Interested diagnostic data may be lost in the noise removing process. To remove the speckle noise, the filter design is difficult in restoration process.

\section{Proposed Method}

The quality of the image is greatly diminished by noise in image acquisition and transmission due to atmospheric turbulence effect, improper usage of sensors in acquisition systems, unknown channel characteristics, motion of the image etc. Such diminished image effectively recovered by the nonlinear image restoration methods. Image restoration techniques are classified as liner and nonlinear upon the mathematical characteristics of degradation model. Here we are using an adaptive filter in noise estimation and restoration process. Adaptive filters are the self aligning filters. Adaptive algorithms [2] are used to adjust the adaptive filter coefficients until to get the minimum mean square error. A kalman filter is simply an optimal recursive adaptive algorithm means that there is no need to store all previous measurements. Because kalman filters are recursive in nature these are continuously time updated for measurement and measurements updated as time instants .By using the kalman filter the entire restoration process changed in to state space model.
The structure of adaptive algorithm using recursive method for image restoration modeled in Figure 2. Here kalman filter is used for modeling of noisy channel (unknown system).This architecture having 2 phases in process, one is unknown channel or system identification and another one is reconstruction process.

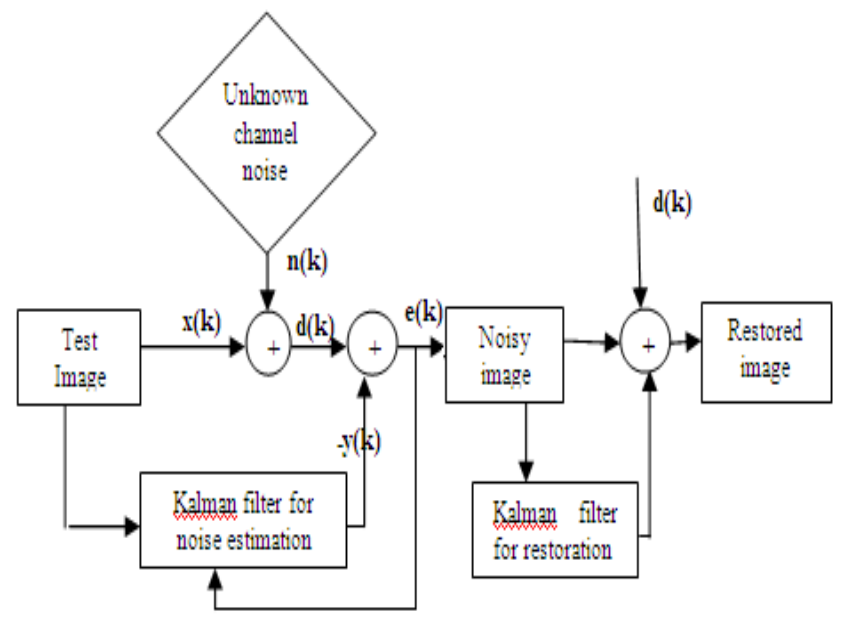

Fig 2. Architecture of image restoration using kalman filter.

The original image $x(k)$ is corrupted by the channel noise $\mathrm{n}(\mathrm{k})$ in transmission or image acquisition process and the desired image $\mathrm{d}(\mathrm{k})$. Where $\mathrm{y}(\mathrm{k})$ in fig 2 representing the noise estimated image . Desired signal at the output of the channel is $\mathrm{d}(\mathrm{k})=\mathrm{x}(\mathrm{k})+\mathrm{n}(\mathrm{k})$ and error signal with noise estimation is $\mathrm{e}(\mathrm{k})=\mathrm{d}(\mathrm{k})-\mathrm{y}(\mathrm{k})$. If the noise is modeled exactly by the kalman filter, adaptive filter will converge immediately then the restored image becomes equal noise free test image. The Kalman filter is a set of mathematical equations that has to be implemented in two steps i.e., a predictor and corrector type estimator steps to minimize the estimated error covariance.

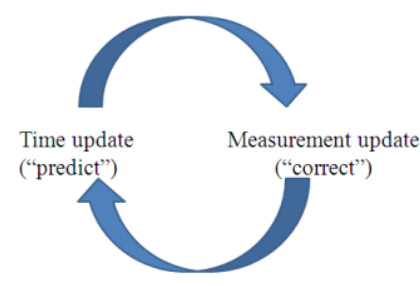

Fig 3. Kalman filter cycle

The time update represents the current state estimate ahead in time. The measurement update adjusts the projected estimate by an actual measurement at that time. The covariance matrix is continuously updated as per the filter cycle. Using kalman filter [3] a random process can be modeled as state model and measurement model.

State model: $\mathrm{x}_{\mathrm{k}+1}=\mathrm{A}_{\mathrm{k}} \mathrm{x}_{\mathrm{k}}+\mathrm{w}_{\mathrm{k}}$ )

Measurement model: $\mathrm{z}_{\mathrm{k}}=\mathrm{hx}_{\mathrm{k}}+\mathrm{v}_{\mathrm{k}}$

Where,

$\mathrm{x}_{\mathrm{k}}=$ state vector

$\mathrm{A}_{\mathrm{k}}=$ state transition matrix

$\mathrm{Z}_{\mathrm{k}}=$ Measurement vector 
$\mathrm{H}_{\mathrm{k}}=$ measurement transition matrix

$\mathrm{v}_{\mathrm{k}}=$ measurement error

The algorithm steps are briefly mentioned in fig 4 in time update and measurement update cycles, resulting that the covariance matrix updated continuously until filter convergence. Because of this covariance updating process there is no need of any memory to store the previous estimations. But the computational complexity increases.

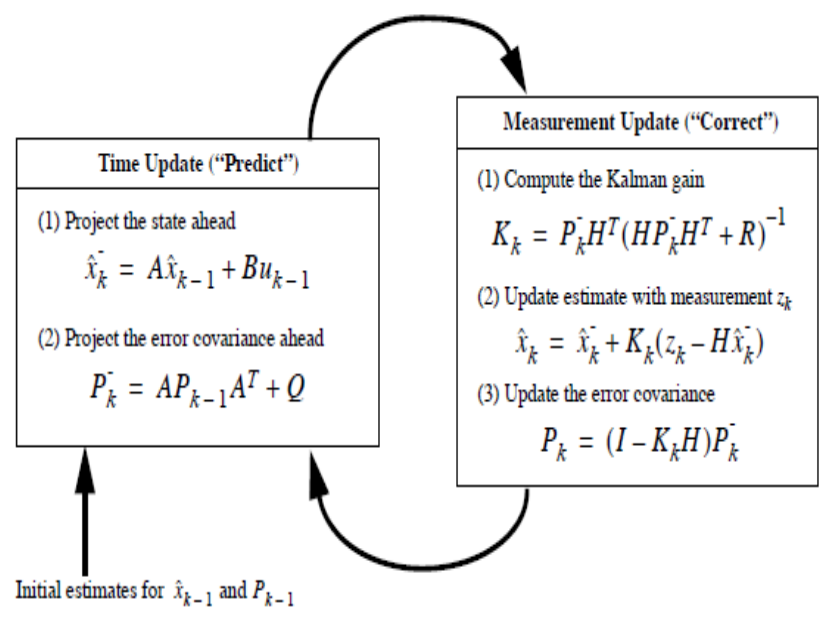

Fig 4. Kalman filter algorithm steps

The quality of the restored image is measured quantitatively interms of MSE, PSNR \&SSIM parameters.

Mean Square Error (MSE): If the original image is equal to the restored image MSE value becomes zero.

$$
\text { MSE }=\frac{1}{M N} \sum_{y=1}^{M} \sum_{y=1}^{M}[f(x, y)-f(x, y)]^{2}
$$

Where, $f(x, y)$ is an noise free input image with the size of $\mathrm{MxN}$, and $x$ and $y$ describes the discrete coordinates of image . $\hat{f}(x, y)$ be the restored image.

Peak Signal to Noise Ratio (PSNR) is the measure of image corrupted by impulse noise, Gaussian noise, and speckle noise .PSNR expressed in logarithmic decibel scale.

$$
\text { PSNR }=10 \log _{10}\left(\frac{255^{2}}{\text { MSE }}\right)
$$

Structural Similarity Index (SSIM) is measuring the degree of similarity between input image and restored image.

$$
\sin =\frac{\left(2 \mu_{x y} \mu_{y}+c_{1}\right)\left(2 \sigma_{z y}+c_{2}\right)}{\left(\mu_{w}^{2} \mu_{y}^{2}+c_{1}\right)\left(\sigma_{z} \sigma_{y}+c_{1}\right)}
$$

\section{RESULTS AND DISCUSSION}

The goal of this experiment is to enhance the quality of medical image subjectively using kalman filter for image restoration. Here considered that the images are corrupted by various noises like Gaussian noise, speckle noise and impulse noise in acquisition and transmission process. The initial condition for degradation filter operation is that the required covariance matrix is assumed based on primitive knowledge of the image, then after the covariance matrix is continuously updating by time updated for weight estimation and measurement estimate for state estimation steps and finally converges to estimate the exact degradation model. Proposed image restoration method is implemented on MAT lab platform. The variance $(\sigma)$ of noise is different for various noises. so the performance of the proposed method is analyzed at different $\sigma$ values from 0.01 to 0.09.The denoising capability of Kalman filter for image restoration is verified quantitavely based on MSE, PSNR, and SSIM parameters and results shown in Table 1.

Table I Quality Assessments of restored medical image

\begin{tabular}{|c|c|c|c|}
\hline Parameters & $\begin{array}{c}\text { Image corrupted } \\
\text { by Gaussian } \\
\text { noise }\end{array}$ & $\begin{array}{c}\text { Image } \\
\text { corrupted by } \\
\text { Salt\& } \\
\text { pepper noise }\end{array}$ & $\begin{array}{c}\text { Image } \\
\text { corrupted by } \\
\text { speckle noise }\end{array}$ \\
\hline MSE & 0.0063 & 0.0030 & 0.0091 \\
\hline PSNR & 80.1798 & 73.2918 & 78.3090 \\
\hline SSIM & 0.9967 & 0.9978 & 0.9984 \\
\hline
\end{tabular}

To demonstrate how kalman filter is suitable for estimation of noise in image restoration we conducted an experiment and those results are summarized in figure 5 .The test image Fig 5(a) taken from medical image database. Various noisy images followed by proposed method result is observed in Fig $5(\mathrm{~b}),(\mathrm{c}),(\mathrm{d})$.

(a)

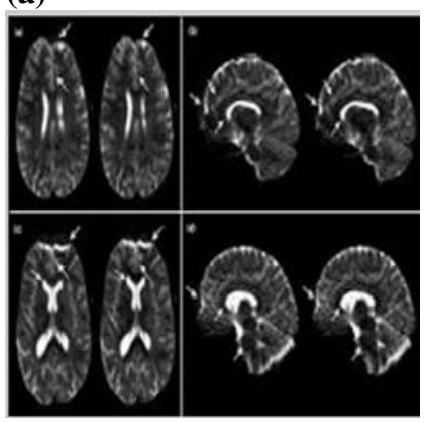

(b)
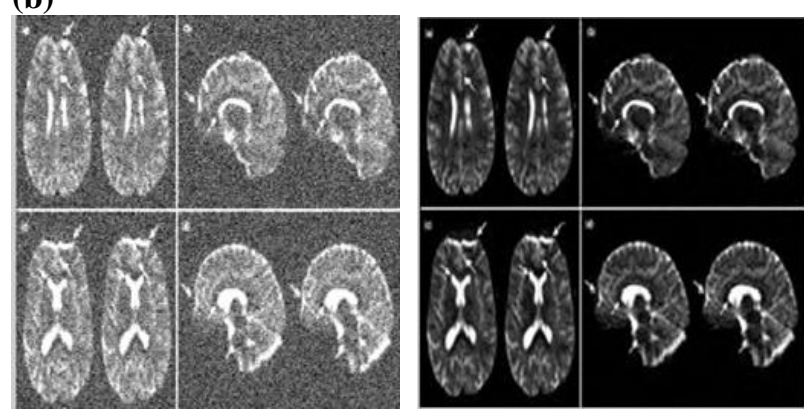

(c)
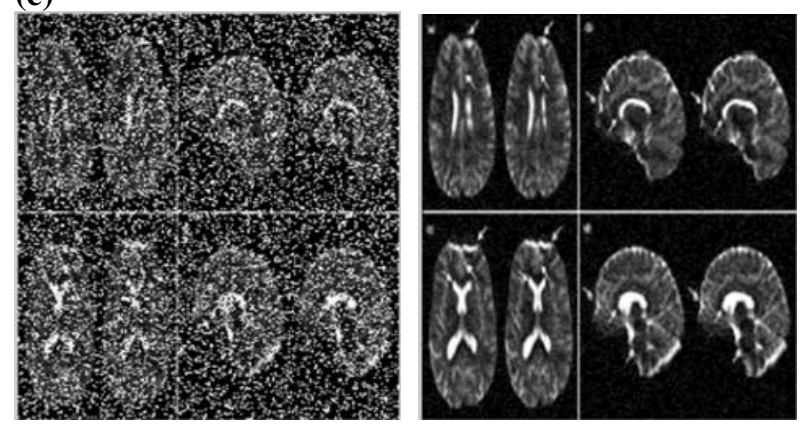

Published By:

Blue Eyes Intelligence Engineering \& Sciences Publication 
(d)
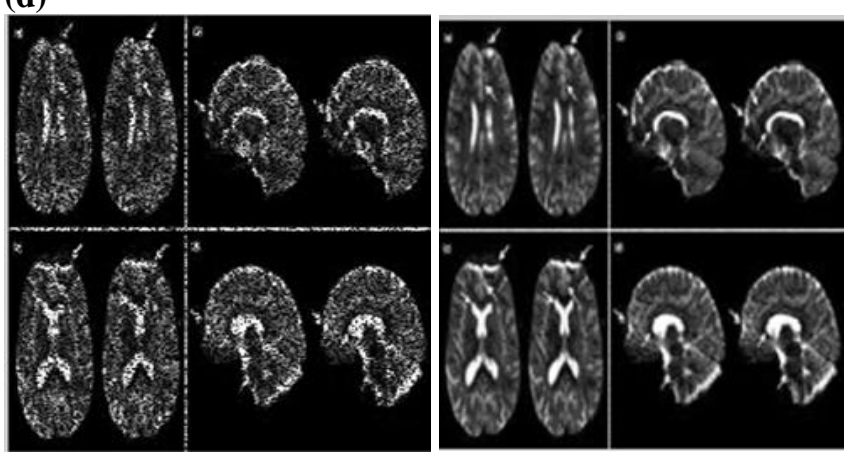

Fig. 5. Medical image restoration results; a) Test image without noise; b) Gaussian noisy image and restored output image(right) ; c) salt and pepper noisy image and restored output image (right) ; d) spekle noisy image and restored output image(right) ;

In this work we evaluated the MSE, PSNR, SSIM parameters of grey scale medical image and observed that the kalman filter improves the quality of image by preserving the edge information.

\section{CONCLUSION}

Here the work carried out only on grayscale medical images which are corrupted by Gaussian noise, salt and pepper noise and speckle noises. But in practice medical images are affected by blur and different artifacts. Colour image processing is the more thrust area in medical applications. Hence more research work required to implement efficient and optimistic restoration techniques for color images.

\section{REFERENCES}

1. O. Sayadi and M. B. Shamsollahi, "ECG denoising and compression using a modified extended kalman filter structure", IEEE Trans. Biomed. Eng, vol. 55, no. 9, (2008), pp. 2240-2248.

2. G. Gui, L. Dai, S. Kumagai, and F. Adachi, "Variable earns profit: Improved adaptive channel estimation using sparse VSS-NLMS algorithms," in IEEE International Conference on Communications (ICC), Sydney, Australia, 10-14 June 2014, pp. 4390- 4394.

3. C. K. Chui and G. Chen, Kalman Filtering, 2nd ed. Berlin, Germany: Springer-Verlag, 1990.

4. Rafael C .Gonzalez and Richard E. woods, "Digital Image Processing", Pearson Education, Second Edition, 2005

5. A. Bouhamidi, K. Jbilou and S. Tikhonov. "Regularization methods in image restoration [J]", Journal of computational and applied mathematics, vol. 206, no. 1, (2007), pp. 86-98.

6. Rinku Kalotra , Sh. Anil Sagar, A Review: A Novel Algorithm for Blurred Image Restoration in the field of Medical Imaging, International Journal of Advanced Research in Computer and Communication Engineering Vol. 3, Issue 6, June 2014.

7. Dougherty G. (2010) "Digital Image Processing for Medical Applications," second ed., Cambridge university press.

\section{AUTHORS PROFILE}

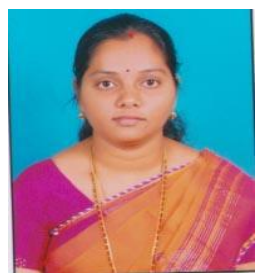

B.Nagasirisha, is a research Scholar, pursuing $\mathrm{Ph}$. D from Jawaharlal Nehru Technological University, Kakinada, Andhra Pradesh, India. She has been working as an Assistant Professor in Gudlavalleru Engineering College, Andhrapradesh, India.

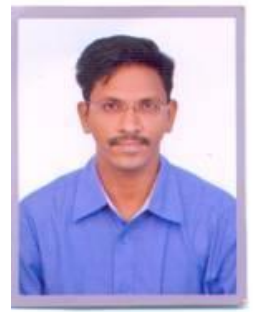

V.V.K.D.V. Prasad, working as a Professor and Head of the Department of Electronics \& Communication Engineering, in Gudlavalleru Engineering College, an Autonomous NBA accredited College in Andhra Pradesh, India. He received $\mathrm{Ph}$. D for his work in Signal Processing in 2011 from Jawaharlal Nehru technological University, Kakinada, India. His areas of interest include Signal Processing, Electrostatics, Electromagnetic fields and Transmission lines. 